\title{
¿De la Frustración a la Alegría o de la Alegría a la Frustración? Significados Sobre Inclusión Educativa Construidos por Docentes a Partir de su Experiencia Emocional
}

\section{From Frustration to Joy or From Joy to Frustration? Meanings About Educational Inclusion Constructed by Teachers Based on Their Emotional Experience}

\author{
Octavio Poblete-Christie \\ Universidad de Playa Ancha \\ Mauricio López y Loreto Muñoz \\ Universidad de Chile
}

\begin{abstract}
El objetivo de esta investigación fue analizar a partir de la experiencia emocional los significados construidos por los docentes sobre la tarea de dar respuesta a la diversidad. Para ello se analizaron 12 entrevistas realizadas a una muestra intencionada, integrada por duplas de profesores regulares y de educación especial que trabajaban conjuntamente en el marco del Programa de Integración Escolar en 4 establecimientos de la Región Metropolitana, Chile. En dichas entrevistas se les pidió a los docentes que narraran incidentes vividos durante la tarea de brindar respuesta educativa a la diversidad y en los que hubiesen experimentado alguna emoción. Se realizó análisis de contenido en base a la teoría fundamentada. Los resultados muestran que los profesores refieren diversas emociones, pero principalmente frustración y alegría. Los significados generados aluden principalmente al logro pedagógico y al ajuste del comportamiento al contexto escolar. Se discute sobre la tensión entre estos resultados y los principios de la educación inclusiva, y sobre las posibilidades que surgen de considerar las emociones como recurso para la comprensión y abordaje del desafío de avanzar hacia una educación más inclusiva.
\end{abstract}

Palabras clave: significados, emociones, inclusión, profesor, entrevista episódica

\begin{abstract}
This study was aimed at analyzing, based on the participating teachers' emotional experience, the meanings constructed by them regarding the task of responding to diversity. To do this, the researchers analyzed 12 interviews with a purposive sample of teacher duos composed of a regular teacher and a special education teacher working together within the framework of the School Integration Program in 4 schools of the Metropolitan Region, Chile. In the interviews, teachers were asked to narrate incidents experienced as part of the task of providing an educational response to diversity and in which they had also experienced emotion. Content analysis was performed based on the grounded theory. Results show that teachers report a variety of emotions, with frustration and joy being the most frequent. The meanings generated mainly allude to pedagogical achievement and to the adjustment of behavior to the school context. The conflict between the results obtained and the guiding principles of inclusive education is discussed, along with the possibilities arising from considering emotions as a resource for understanding and tackling the challenge of achieving a more inclusive education.
\end{abstract}

Keywords: meanings, emotions, inclusion, teacher, episodic interview

Con el propósito de avanzar en materia de inclusión educativa, en los últimos años se han desarrollado reformas que buscan incidir en las prácticas pedagógicas y las culturas escolares. Sin embargo, algunas de estas políticas han sido caracterizadas como contradictorias, híbridas o antagónicas. Refiriéndose a la situación internacional, Slee (2011/2012) ha planteado que la "política laberíntica" que trata de hacer frente a la exclusión educativa presenta múltiples niveles de tensión política y presiones contradictorias que terminan muchas veces en la continuación de prácticas antiguas bajo la capa de un nuevo léxico. Las

Octavio Poblete-Christie, Departamento de Educación, Universidad de Playa Ancha, San Felipe, Chile; Mauricio López y Loreto Muñoz, Departamento de Psicología, Universidad de Chile, Santiago, Chile.

Los datos analizados en este artículo forman parte del Proyecto FONDECYT No 11110141 , "Inclusión educativa en Chile. Recursos y barreras de los procesos de mejora de la atención a la diversidad a través de un estudio de casos de establecimientos educacionales municipales con proyectos de integración", realizado entre el periodo 2011 y 2014 y cuyo investigador responsable fue el Dr. Mauricio López Cruz.

La correspondencia relativa a este artículo debe ser dirigida a Octavio Poblete Christie, Departamento de Educación, Universidad de Playa Ancha, Sargento Luis Navarrete 1981, Nuñoa, Santiago, Chile. E-mail: octavio.poblete@gmail.com 
ambigüedades y tensiones actuales del campo de la educación inclusiva pueden deberse, según Norwich (2014), al hecho de asumir para las políticas y las prácticas escolares una serie de valores cuyo significado es a veces confuso, tales como equidad, calidad, justicia social, democracia, participación y diversidad. En Chile, López, Julio, Morales, Rojas y Pérez (2014) señalan que la política de integración escolar de los últimos años presenta una "hibridez paradigmática", puesto que "convoca a la inclusión desde la lógica de los derechos humanos, pero prescribe un modelo de integración con un fuerte énfasis psico-médico con diagnósticos y tratamientos pedagógicos individuales a través de la figura de la docente especialista" (p. 256). En este trabajo se entendió inclusión educativa como el proceso mediante el cual el sistema educativo ofrece respuestas pedagógicas a las diferentes necesidades e identidades de los estudiantes, garantizando el derecho a la educación de todos. En este sentido, es necesario puntualizar que, desde esta perspectiva, los sujetos de inclusión son todos los estudiantes y no un grupo específico identificado con algún diagnóstico o rasgo identitario en particular.

En este escenario, la complejidad del aula plantea, pues, una serie de desafíos en el ámbito relacional a los profesores. Por ejemplo, a partir de la entrada en vigencia del Decreto 170 del Ministerio de Educación de Chile (el que regula las subvención escolar para el funcionamiento de los programas de integración escolar), se plantea una nueva relación entre los profesores de educación general y especial, la que requiere renegociar los significados asociados a cada una de estas culturas profesionales (Muñoz Villa, López Cruz \& Assaél, 2015). Así también, al reflexionar sobre los apoyos relevantes para el aprendizaje de los estudiantes que enfrentan mayores dificultades, los docentes consideran el apoyo afectivo que les proporcionan a éstos como una herramienta pedagógica eficaz, pero difícil de poner en práctica en las actuales condiciones de trabajo (Muñoz Villa et al., 2015). En este sentido, Echeita, Sandoval y Simón (2016, Marzo) concluyen que profesores y profesoras tratan de conectar imaginativa y empáticamente con sus estudiantes, buscando comprender los acontecimientos del aula desde las acciones, elecciones, emociones, atribuciones y expectativas de sus estudiantes.

A su vez, la valoración intrínseca de los seres humanos y el cuidado como ética fundamental de la relación pedagógica aparecen como aspectos moduladores de la inclusión (Urbina Hurtado, 2013), pues es en la dimensión emocional de la inclusión donde se ponen en juego aspectos tales como la disposición a entregar apoyo, la aceptación y el rechazo, la confianza y la desconfianza (Urquhart, 2009), así como también el establecimiento de una convivencia respetuosa, solidaria y digna (Echeita, Simón, López \& Urbina, 2013). Del mismo modo, parece claro para muchos que el vínculo afectivo y el apoyo moral entre profesores y estudiantes son facilitadores de la inclusión (López Cruz, Valdivia Barrios \& Fernández Droguett, 2016; Torres et al, 2013) y, por el contrario, que la discriminación ejerce efectos dañinos sobre el autoconcepto y la motivación de los estudiantes (Urquhart, 2009).

Más concretamente, algunos estudios han abordado la dimensión emocional en la respuesta educativa a estudiantes con características específicas. Por ejemplo, Poulou y Norwich (2002) desarrollaron un modelo predictivo de la respuesta de los profesores hacia estudiantes con problemas emocionales y de conducta, concluyendo que su disposición a ayudarlos está mediada por la atribución de causalidad de las dificultades del niño y la emoción que los propios niños despiertan en ellos. También, un trabajo reciente realizado en Chile con profesores que trabajan con estudiantes con trastornos del espectro autista permitió reconocer la relevancia de la afectividad como un recurso pedagógico para la comunicación con estos estudiantes (ZentenoOsorio \& Leal-Soto, 2016).

En términos más amplios, Hargreaves (2000) ha analizado la complejidad del cambio educativo, incorporando el papel que juegan las emociones en el quehacer docente. Para ello, ha recurrido al concepto de geografías emocionales, es decir, patrones espaciales y experienciales de cercanía y distancia presentes en la interacción humana que permiten crear, cualificar y comprender las relaciones con uno mismo y con los demás. Asimismo, ha reparado en la presencia de una "política emocional" perpetuadora del fracaso escolar que, al focalizarse en las escuelas que obtienen malos resultados, terminan reproduciendo y perpetuando la desigualdad social vinculada a dichos resultados, generando de manera diferencial emociones de distinción y orgullo en los estudiantes que tienen éxito y de disgusto y vergüenza en quienes fracasan (Hargreaves, 2003).

Tomando en cuenta la complejidad presente en el estudio de las emociones, el trabajo realizado por Golombek y Doran (2014) sobre formación docente resulta interesante pues, inspirándose en la conceptualización de Vygotsky, estudian el papel de las emociones sobre el aprendizaje, considerándolas como parte de una sola unidad de análisis, junto con el pensamiento y la acción. Efectivamente, el enfoque histórico cultural rechaza el intento tradicional de "trazar fuertes límites conceptuales entre el pensamiento, la emoción y la acción” (Bruner, 1985/1996, p. 112). Por otra parte y sin descartar la base biológica de las 
emociones, esta perspectiva enfatiza la programación o rúbrica que la cultura realiza sobre estas (Bruner, 1985/1996). Es así como, por ejemplo, es posible comprender la presencia de diferencias sustantivas entre las poblaciones estadounidense y china en relación a la experiencia de la vergüenza (Mascolo, Fischer \& Li, 2003).

A su vez, las impresiones de naturaleza afectiva que los seres humanos desarrollan producto de sus encuentros con el mundo cumplen un rol activo en la construcción de su realidad (Bruner, 1985/1996). De ahí que, posiblemente, se entienda que la emoción surja justamente cuando "una manera de construir el mundo está fuera de control" (Bruner, 1985/1996, p. 116). La construcción de la realidad social y el surgimiento de emociones estarían íntimamente articulados, existiendo, por tanto, una relación estrecha entre estas y los significados que se negocian en el contexto cultural, proceso que, a su vez, actúa sobre este último y lo configura.

Los significados actúan como articuladores entre el individuo y el entorno cultural y son producidos a través de narraciones. Fruto de la interacción, los miembros de una determinada comunidad construyen versiones normativas de la realidad que les permiten interpretar el mundo, las cuales, por otra parte, están sometidas a un permanente proceso de negociación. Es decir, los significados se expresan y se transforman cuando las personas negocian sus nociones de mundo a través de relatos que intercambian con otros actores. Además, la efectividad de las narraciones dependería de la presencia de los siguientes aspectos gramaticales: agentividad, es decir, la presencia de acción intencionada de un sujeto dirigida hacia una meta o propósito; secuencialidad, dada por el orden consecutivo de los hechos narrados; sensibilidad hacia aquello que resulta acostumbrado e inusual en una cultura y subjetividad involucrada, es decir, la manera particular de interpretar los hechos por parte de quien los narra (Bruner, 1990/1991).

De esta forma, en el camino de transformación cultural que supone dar respuesta a la diversidad de necesidades e identidades de los estudiantes, resulta de interés conocer los significados de los docentes sobre el trabajo que realizan en el contexto de prácticas situadas, puesto que de esa forma sería posible acceder tanto a su propia subjetividad como a la comprensión de los aspectos culturales actualmente presentes en tales escenarios, todo lo cual permitiría proporcionar pistas sobre la dirección de las acciones para conseguir los objetivos deseados. Asimismo, resulta razonable estimar que la indagación sobre los significados producidos a partir de situaciones que gatillan emociones resulta relevante de explorar, dada la estrecha conexión de estos estados mentales con la realidad construida culturalmente (Bruner, 1985/1996) y, también, dada la valencia, espontaneidad y ausencia de control consciente con que estos surgen (Aguado, 2005). Además, y tal como se señalaba desde un inicio, una aproximación de este tipo permitiría acceder a la dimensión axiológica constitutiva de la inclusión educativa (López, Echeita \& Martín, 2010).

El presente artículo corresponde a un análisis complementario de un estudio ya publicado (Muñoz Villa et al., 2015), cuyo objetivo era examinar, a partir del relato de episodios críticos, las concepciones de profesores regulares y de apoyo sobre algunas dimensiones de la inclusión educativa. El presente trabajo, en tanto, tuvo como objetivo profundizar, a partir del mismo material, en el modo en que se elaboran significados respecto a la tarea de brindar respuestas educativas a la diversidad, poniendo el foco en las emociones que suscitan dichos episodios y cómo estas se integran en la experiencia del docente.

\section{Método}

\section{Participantes}

La muestra fue intencionada y estuvo conformada por informantes clave. La elección de los niveles educativos obedeció al criterio elaborado con los equipos de las escuelas, referentes al mayor desafío que implicaba la respuesta a la diversidad en dichos niveles. Participaron 24 profesores (20 mujeres y 4 hombres), cuyas edades fluctuaban entre 25 y 60 años. Dieciocho se desempeñaban en segundo ciclo básico ( $5^{\circ}$ a $8^{\circ}$ básico) y seis en enseñanza media ( $1^{\circ}$ a $4^{\circ}$ medio). Las entrevistas se organizaron en duplas, compuestas por un docente de apoyo y un docente de educación regular que trabajaban conjuntamente en aula. Estos pertenecían a cuatro establecimientos de dependencia municipal, tres de educación básica y un liceo de enseñanza media de la Región Metropolitana de Chile, los que contaban con un Programa de Integración Escolar (PIE). En cada establecimiento se entrevistó a tres duplas. La entrevista fue realizada por una profesora de educación diferencial, magíster en psicología educacional. 


\section{Técnicas de Producción de Datos}

Los datos fueron obtenidos a través de entrevista episódica. Este tipo de técnica proporciona elementos provenientes de dos tipos de actividades mentales diferentes: el conocimiento narrativo episódico y el conocimiento semántico. El primero está asociado estrechamente a las experiencias vividas en situaciones específicas y el segundo se relaciona con abstracciones que se realizan de tales experiencias (Flick, 2002/2007). Las entrevistas se realizaron en base a episodios correspondientes a situaciones en las que ellos, de manera conjunta o no, se vieron enfrentados a tener que dar respuesta educativa, en forma individual y/o colaborativa, a necesidades individuales de estudiantes específicos (que podían o no ser parte del PIE) en el contexto escolar. Además, el episodio debía haber causado alguna emoción, por considerarlo difícil, conflictivo, exitoso o esperanzador. Así, los episodios se definieron como eventos vividos en el contexto educativo en que: el docente se implicaba personalmente, tenían una duración acotada en el tiempo y en el espacio, y en los que participaban distintos actores, tales como profesores, estudiantes o apoderados. La naturaleza narrativa de los datos producidos fue particularmente pertinente para este trabajo, puesto que esta se considera primordial para indagar los significados construidos en un determinado contexto (Bruner, 1990/1991).

En estas entrevistas participaron simultáneamente ambos miembros de cada dupla, mediante la escucha de su colega y la participación como co-entrevistador, lo cual permitió potenciar el carácter dialógico y reflexivo de la técnica. Este proceso pretendía que los entrevistados reflexionaran conjuntamente sobre sus prácticas, identificaran su experiencia subjetiva, levantaran hipótesis y generaran una racionalidad alternativa para la acción.

\section{Procedimiento}

Las entrevistas fueron registradas en audio y posteriormente transcritas. Los participantes firmaron un formulario de consentimiento informado, a través del cual fueron debidamente informados de los objetivos, metodología y confidencialidad de los datos, dejando estipulado el carácter voluntario de su participación.

\section{Análisis de Datos}

Se realizó análisis de contenido en base a la teoría fundamentada (Flores \& Naranjo, 2013; Strauss \& Corbin, 1998/2002). Para ello, se utilizó el programa ATLAS.TI.

En primer lugar, se identificaron 31 episodios. En 23 de ellos los actores refirieron haber experimentado al menos una emoción y en ocho, ninguna. Luego, se codificaron las transcripciones, en base a lo que se denominó códigos en vivo (Albornoz, Silva \& López, 2015), mediante lo cual se redujeron gramaticalmente expresiones significativas que daban cuenta de emociones experimentadas por los participantes durante los episodios, manteniendo, así, para el análisis posterior las propias palabras de los participantes. En esta codificación se incluyeron, además, expresiones relacionadas con la empatía y la simpatía, considerando su estrecha relación con las emociones. Durante este proceso, se tomaron en cuenta no solo vocablos convencionales para consignar las expresiones referidas a emociones ("sentí rabia"), sino también expresiones afines, por ejemplo, "sentí molestia" (rabia, enojo), "qué rico" (agrado, satisfacción) y "te pasan cosas re bonitas" (agrado, satisfacción, alegría). Asimismo, se seleccionaron referencias que utilizaban expresiones coloquiales o modismos (p. ej., "me vino una depre [depresión]" o "me da pica [rabia]"), o metáforas (p. ej., "esto es como darse contra la pared"). En total, se generaron 98 códigos en vivo. Los sistemas de códigos se sometieron a un proceso progresivo de triangulación entre los autores del estudio, siguiendo el argumento de Miles y Huberman (1994), quienes puntualizan que los sistemas de categorías se configuran como "subculturas" creadas mediante chequeos periódicos y continuos de fiabilidad entre investigadores que desarrollan su propia lengua "vernácula". Por lo tanto, "los datos codificados no tienen significado en sí mismos, sino que son el resultado del proceso gradual de construcción de sentido del propio equipo investigador" (Miles \& Huberman, 1994, p. 89).

En segundo lugar, los códigos seleccionados fueron agrupados en virtud de su conexión con emociones reconocidas como tales por la literatura, tomando como punto de partida intentos de clasificación más bien clásicas, como las de Ekman, Friesen \& Ellsworth (1982) o Izard (1977), hasta aquellas concepciones de emoción más amplias y culturalistas (Belli, 2009), procurando en todo caso que las citas reflejaran de alguna forma una sensación experimentada por los entrevistados en un lapso acotado de tiempo (Barrett \& Russell, 2015; Oatley, 1992). Es así como se fue logrando una coincidencia entre códigos en vivo y códigos conceptuales (Flores \& Naranjo, 2013), tal como se ejemplifica en la Tabla 1. 
Tabla 1

Ejemplos de Codificación

\begin{tabular}{|c|c|}
\hline Código en vivo & Código conceptual \\
\hline Me siento contenta y satisfecha cuando salgo de una clase que resultó & Alegría \\
\hline $\begin{array}{l}\text { Siento orgullo de trabajar con profesores que nos damos al } 100 \% \text { a nuestras } \\
\text { alumnas }\end{array}$ & Orgullo \\
\hline Me llega lo que el otro siente & Empatía \\
\hline
\end{tabular}

A continuación, se elaboró una caracterización de cada uno de los episodios, que consideró un breve resumen de cada uno, el tipo de actor con quien interactuaba el narrador de cada episodio (personaje) y las emociones del narrador asociadas a cada uno de esos episodios (Tablas 2 y 3 ).

Tabla 2

Características de los Episodios Narrados por Profesor Regular

\begin{tabular}{rlll}
\hline $\mathrm{N}^{\circ}$ & \multicolumn{1}{c}{ Breve descripción } & \multicolumn{1}{c}{ Personaje } & \multicolumn{1}{c}{ Emociones } \\
\hline 1. & Estudiante se arranca de la sala & Estudiante & Rabia \\
2. & Estudiante que abandona el establecimiento & Estudiante & Alegría, rabia \\
3. & Logros de estudiante con discapacidad intelectual & Estudiante & Frustración, empatía, alegría \\
4. & Estudiante con rendimiento bajo & Estudiante & Empatía, tranquilidad, miedo \\
5. & $\begin{array}{l}\text { Proceso de trabajo con estudiante diagnosticado con } \\
\text { síndrome de Asperger }\end{array}$ & Estudiante & Tranquilidad, gratificación, humor, \\
miedo, empatía
\end{tabular}

Tal como se puede observar en las Tablas 2 y 3, a lo largo de los diferentes episodios narrados se identificaron 13 emociones: seis fueron de valencia positiva (alegría, agrado, satisfacción, gratificación, tranquilidad, humor) y siete de valencia negativa (frustración, rabia, miedo, preocupación, nerviosismo, pena y dolor). Además, se consideró la referencia a situaciones de empatía y de simpatía. Del extenso material obtenido en esta etapa, se seleccionó aquel que daba lugar a las categorías frustración y alegría, ya que fueron las que concentraban mayor número de citas. Sobre estas categorías, se realizó un análisis detallado en torno a los significados construidos por los participantes. Cabe destacar que en el análisis de los significados surgidos en relación a la alegría se consideraron también emociones afines (agrado, satisfacción y gratificación) y, en el caso de la frustración, se consideró también la rabia, dada la relación estrecha entre ambas (Kamenetzky et al., 2009). 
POBLETE-CHRISTIE, LÓPEZ Y MUÑOZ

Tabla 3

Características de los Episodios Narrados por Profesor de Apoyo

\begin{tabular}{|c|c|c|c|}
\hline $\mathrm{N}^{\circ}$ & Breve descripción & Personaje & Emociones \\
\hline 1. & $\begin{array}{l}\text { Satisfacción por logros de aprendizaje en lectura } \\
\text { estudiante sordo }\end{array}$ & Estudiante & Simpatía, satisfacción, empatía \\
\hline 2. & $\begin{array}{l}\text { Avances significativos de estudiante con problemas } \\
\text { psicomotores }\end{array}$ & Estudiante & Agrado, alegría \\
\hline 3. & Avances socioafectivos logrados con un estudiante & Estudiante & $\begin{array}{l}\text { Agrado, tranquilidad, alegría, rabia, } \\
\text { preocupación, empatía }\end{array}$ \\
\hline $4 .$. & $\begin{array}{l}\text { Logros obtenidos con apoyo familiar en estudiante con } \\
\text { discapacidad intelectual }\end{array}$ & Estudiante & Alegría \\
\hline 5. & $\begin{array}{l}\text { Estudiante diagnosticada con trastorno específico de } \\
\text { aprendizaje que no avanza }\end{array}$ & Estudiante & Frustración \\
\hline $6 .$. & $\begin{array}{l}\text { Proceso con estudiantes diagnosticados con trastorno por } \\
\text { déficit atencional }\end{array}$ & Estudiante & $\begin{array}{l}\text { Agrado, tranquilidad, humor, } \\
\text { frustración, empatía }\end{array}$ \\
\hline 7.. & $\begin{array}{l}\text { Profesora es acusada de maltrato a estudiante con } \\
\text { discapacidad física }\end{array}$ & Apoderado & Frustración (2), dolor, empatía \\
\hline 8. & Profesores regulares critican a educadora de apoyo & Profesor & Frustración (3), preocupación \\
\hline 9. & $\begin{array}{l}\text { Estudiantes son presionados a abandonar el } \\
\text { establecimiento }\end{array}$ & $\begin{array}{l}\text { Miembro del equipo } \\
\text { directivo }\end{array}$ & $\begin{array}{l}\text { Preocupación, frustración, } \\
\text { nerviosismo, pena }\end{array}$ \\
\hline
\end{tabular}

\section{Resultados}

En primer lugar, se exponen los significados relacionados con la frustración y, a continuación, los significados relacionados con la alegría. Para cada fragmento de entrevista se indica el número de la entrevista, el número de línea asignado al documento primario respectivo, el tipo de profesor y nivel educativo correspondiente.

\section{Significados Asociados a la Frustración}

En comparación con todas las otras emociones reportadas durante las entrevistas los docentes profundizaron de manera especial en la frustración. En el análisis de los significados surgidos en relación a esta emoción se consideró también la rabia, dada la relación estrecha entre ambas (Kamenetzky et al., 2009). A continuación se presentan estos resultados organizados en función de los códigos en vivo elaborados durante el proceso análisis.

Me frustra no lograr avances con los alumnos. La dificultad para lograr avances en estudiantes con quienes se trabaja de manera directa es referida reiteradamente como causa del surgimiento de emociones de frustración. Dicha dificultad ocurre en relación a resultados de aprendizaje, aunque también en relación a la motivación de los estudiantes. Algunos datos apuntan a aspectos de orden individual o intraindividual, profundizando en los significados que los docentes construyen sobre su experiencia emocional propiamente tal. En cambio, otros datos, que se analizarán más adelante, se focalizan en aspectos de orden interpersonal y contextual. Los datos que profundizan en la experiencia individual adoptan significados diversos. A veces, la frustración experimentada es significada como fruto de una limitación de las propias capacidades:

Porque yo trato de motivarlos con que es $8^{\circ}$, con que hay que salir de $8^{\circ}$, que queda poco, que vamos, pero ni eso. Entonces, siento que se me agotan un poco las estrategias de motivación de ellos. (entrevista 7, 222, profesora de apoyo, enseñanza básica)

Otras veces, la frustración es explicada a partir de la pérdida de control sobre el grupo curso:

Pero también salgo muy (...) frustrada cuando (...) se han portado pésimo, llegaron buenos para hablar y no pude tomar en ese rato el control de la situación y haber hecho el cambio, también, salgo bien frustrada. (entrevista 7, 107, profesora regular, enseñanza básica).

O bien, en el trabajo individual con un estudiante, la frustración es interpretada simplemente como un obstáculo imposible de franquear:

No había por dónde, porque (...) escribía en carro, yo le decía "escribe bien, está en la pizarra", me sentaba al lado de él... era mirar... muchos detalles que, definitivamente, hacían que no avanzáramos nada. Y yo me sentía 
muy frustrada, porque yo decía que en realidad esto es como darse contra la pared. (entrevista 8, 61, profesora de apoyo, enseñanza básica)

No obstante, es destacable el hecho paradójico de que esta emoción cambie su carga negativa y se transforme en un motor para la enseñanza. Es decir, por una parte, hay un reconocimiento de la valencia negativa involucrada en esta emoción, pero, en un nivel reflexivo superior, dicha experiencia emocional se transforma en motivación que inyecta energía positiva para continuar con el trabajo educativo:

Pero, es como más desafiante, es frustrante, pero es más como un desafío personal. (entrevista 7, 212, profesora de apoyo, enseñanza básica)

Esta frustración, gatillada por la falta de avances, suele ser interpretada más como producto de un fracaso propio que como un problema de los estudiantes, lo cual, además, reflejaría un importante grado de compromiso invertido en el trabajo pedagógico. Y este fracaso es significado no solo en términos profesionales y laborales, sino también en términos personales. A su vez, la estrecha interdependencia reportada entre la evaluación del propio trabajo y los resultados educativos de los estudiantes evidencian el carácter relacional de la labor pedagógica:

Igual yo me siento frustrada un poquito (...) porque esa era parte de mi trabajo (...). Entonces, que ellos (...) no hayan logrado el objetivo que yo quería con ellos, viene siendo para mí parte de mi fracaso. (entrevista 2, 107, profesor de apoyo, enseñanza media)

También la frustración es significada en función de una diferencia con los colegas en la forma en que se evalúan los progresos de los estudiantes. En concreto, se describe cómo el trabajo de una profesora de apoyo fue objeto de cuestionamiento por parte de una profesora regular, sin que esta considerara, en apariencia al menos, las características distintivas de las estudiantes con quienes estaba trabajando:

Y en el hecho de que la profesora alega mucho (...) que las niñas no tienen avance (...) pero igual hay que pensar que son niñas con dificultades, o sea, no van al mismo nivel que sus compañeras (...) mis frustraciones son esas, como que se cuestione mi trabajo como, como educadora, y que uno lo trata de hacer lo mejor posible, pero no son todos los niños iguales y todos aprenden de distinta manera. (entrevista 10, 124, profesora de apoyo, enseñanza básica)

En un nivel diferente, es posible observar que este tipo de frustración no solo surge de una diferencia de criterio pedagógico para responder a las necesidades de los estudiantes, sino que además se experimenta una profunda desconsideración hacia su propio trabajo. Es el caso de la situación que se genera a partir de la presión institucional orientada a lograr que dos estudiantes renunciaran a continuar en el establecimiento. El problema se origina porque las docentes que se encontraban trabajando directamente con tales estudiantes se enteraron de lo ocurrido una vez que los hechos estaban consumados, situación que las sorprendió totalmente, debido a que ellas venían observando una mejoría en el proceso escolar de los estudiantes:

Estaban mejorando, estaban subiendo sus notas [calificaciones], estaban ya dejando de, de hacer lo que normalmente hacían. (entrevista 2, 141, profesor regular, enseñanza media)

Es así como la frustración experimentada ante la noticia de la salida de estos estudiantes es explicada por los profesores implicados no solo por lo tardío del momento en que fueron informados sobre esta situación sino, sobre todo, por la falta de consideración hacia ellos por parte de las autoridades en el momento mismo en que ocurrieron los hechos, impidiéndoles la posibilidad de haber participado y eventualmente incidido en la salida de los estudiantes del establecimiento:

Entonces, creo que por esa parte igual uno se frustra más, porque uno ya está con un trabajo encima, está con un proyecto para ello, tiene a lo mejor otra mirada de su futuro y de un día para otro es que se fue. Entonces, en ningún momento se pide la opinión, en ningún momento se pregunta si está bien lo que están haciendo, la determinación que están tomando. (entrevista 2, 149, profesor de apoyo, enseñanza media)

Me siento frustrada cuando no puedo comunicarme adecuadamente con un alumno. El trabajo educativo es de naturaleza relacional, lo que implica necesariamente un diálogo con los estudiantes y, por lo tanto, la obstaculización a este proceso constituye en sí mismo una fuente de frustración. Esta emoción aflora en una profesora de Lenguaje, al no poder comunicarse con un estudiante, debido a su discapacidad, mermando con ello las posibilidades de vinculación e intercambio pedagógico:

Esa fue la frustración más grande, no poder... que me entendieran, comunicarme, así como me comunico con cualquier alumno normal ¿te fijas? normal entre comillas, son todos normales, sino que por su discapacidad. (entrevista 1, 346, profesora regular, enseñanza media)

En esta referencia aparece la atribución del problema comunicativo a las características del estudiante más que a la ausencia de herramientas o estrategias de la propia profesora para entablar el contacto con el estudiante. 
Por otra parte, aunque de una manera menos directa, las aulas muy pobladas imponen limitaciones al docente que le impiden una comunicación sistemática con cada estudiante:

Me cuestiono y digo "pucha [expresión de molestia], tengo tantas en la sala, que tampoco me puedo enfocar solamente en la Victoria" (...) Estás finalmente como un poco atada de manos, por la cantidad de niñas que hay en la sala. (Entrevista 10, 48, profesora regular, enseñanza básica)

Se observa en los dos fragmentos una preocupación por los obstáculos que se interponen en la comunicación con los estudiantes y que impiden llevar adelante el trabajo pedagógico.

Me da rabia que no se comporten como se debe. La rabia aparece algunas veces ligada a la evaluación que las profesoras realizan de la conducta de sus estudiantes, de los apoderados o de colegas, en base a determinadas normas o valores. De esta forma, esta emoción aparece en la vivencia personal de la profesora cuando el comportamiento de un estudiante no se ajusta al contexto escolar. Por ejemplo, cuando un estudiante se enojaba con sus compañeros, este simplemente se iba intempestivamente de la sala, golpeando la puerta, y se encerraba en el baño. Dicha situación no solo generaba la rabia de una de las profesoras, sino que además activaba en ella cursos automáticos de acción:

Antes se enojaba y se arrancaba y yo lo salía persiguiendo y se encerraba en el baño y yo no podía abrir la puerta por fuera y a mí me daba una pica [rabia] terrible (entrevista 5, 261, profesora de apoyo, enseñanza básica)

La situación descrita se explicaría por la brecha entre la conducta del estudiante y lo que la profesora esperaba de su comportamiento. En otras ocasiones, la frustración también es explicada por un desajuste percibido por la profesora entre el esfuerzo desplegado por ella en la preparación de sus clases y la baja participación de sus estudiantes en estas:

A mí una de las cosas que me, me da, no sé, si rabia, lata, no sé cómo expresarlo, es que yo me saco la mugre [trabajo duro] planificando, revisando esto, haciendo esto, tratando de hacer la clase lo mejor, poniéndome, cuando la estoy planificando, en la situación del niño, cómo la va a entender, todo. Llego a la sala y por más que me falta hacerme de tony [payaso] no más, no consigo nada ¿ya? Entonces, digo yo, “¿qué está pasando con nuestra juventud?" y yo veo que no hay valores. (entrevista 12,119 , profesora regular, enseñanza básica)

A su vez, los valores a los cuales alude la profesora serían propios de otra época, vivida durante su historia como estudiante, y contrastarían con lo que ocurre en los actuales escenarios escolares:

Antes yo recuerdo cuando tú eras lolita [mujer adolescente] o a lo mejor ni existías todavía, po ¿ya? Pero el profesor era autoridad ¿ya? Incluso cuando yo era chica yo me acuerdo que mi profe lo que decía era ley y los papás también decían "sí, po, tu profesora lo dijo" ¿ya? Cuando yo entré a trabajar, también ¿ya? Pero después, actualmente no es así la cosa ¿ya? (entrevista 12, 119, profesora regular, enseñanza básica)

Así, esta frustración se explica a partir de las expectativas no satisfechas que la profesora había construido respecto de sus estudiantes, pero no en función de orientaciones escolares actuales, tal como ocurría en el caso anterior, sino más bien a partir de un escenario valórico pretérito, que persistía como referente en la propia subjetividad de esta profesora.

Por otra parte, este tipo de malestar se origina también a partir de determinadas actitudes de los apoderados, específicamente en relación al supuesto descuido en el ejercicio del rol parental. Se trata de una evaluación realizada por una profesora sobre una interpretación que ella hace de la subjetividad de una apoderada y que apuntaría a su falta de compromiso con el proceso educativo de su hijo. Dicha evaluación es realizada a partir de marcos valóricos socioculturales amplios, sobre el cuidado de los niños y niñas y, en particular, sobre el rol que deben tener los padres y apoderados en la educación de sus pupilos:

Y lo que más me molesta a mí de esta situación es la mamá, que, que no tiene ningún interés en que su hijo viniera a clases. (entrevista 6,78, profesora regular, enseñanza básica)

Finalmente, la rabia aparece en un profesor regular por la percepción de desbalance entre la baja remuneración percibida, en comparación con la de otros colegas, cuyo volumen de trabajo sería mucho menor que el suyo. Además, el abultamiento de dichas remuneraciones contrastaría con la carencia de recursos económicos del establecimiento para afrontar diversas necesidades. En este sentido, la frustración es significada como producto de una injusticia:

Hay tanta gente acá mismo que gana mucha plata por no hacer nada, después dicen "no, es que no hay plata, no hay plata para hacer esto, no hay plata para comprar esto, no hay plata para la implementación de este, de este, de esta sala”, pero para tener gente abajo sentada, que no hace nada, ahí sí (...) Yo... paso corriendo pa' allá, paso corriendo pa' acá y siempre los veo ahí sentados, así... o en la mesa, así, o tomando tecito, su galleta... (entrevista 2, 410, profesor regular, enseñanza media) 
Me frustran las condiciones del entorno. Más allá de las expectativas interpersonales, las condiciones materiales también generan frustración. Es el caso de la falta de infraestructura y materiales debido a la demora en la entrega de los recursos por parte de la Corporación Municipal. En este sentido, hay referencia a lo que podría denominarse como una falta de mutualidad en la relación entre esta instancia y quienes realizan el trabajo pedagógico, en términos de que la Corporación les exige resultados educativos, pero no les entrega el apoyo económico con la celeridad requerida. De esta manera, las profesoras no ven más remedio que realizar un aporte económico personal:

Recursos que no llegan desde donde nos exigen justamente, desde donde nos están exigiendo cumplir con todo, pero ellos no nos surten tampoco. Nosotras, la plata de integración no la vemos desde el año pasado, no vimos nada. El año pasado, todo lo que tuve que hacer en la impresión de los formularios, el informe, salió de nuestro bolsillo. (entrevista 10, 175, profesora de apoyo, enseñanza básica)

Se trata, entonces, de una situación de desigualdad en la relación entre distintos actores involucrados y que provoca emociones de valencia negativa:

Y sabís que ha sido frustrante (...) Y esa... tenía que imprimir unas, unas letras, tenía que imprimir... y a nosotros se nos acaba la tinta, y andamos todos de... se nos acaba la tinta y andamos llorando, andamos todos mal. (entrevista 10, 184, profesora de apoyo, enseñanza básica)

Mi frustración es que me traten injustamente. La percepción de ser tratado injustamente ya ha sido mencionada como motivo de frustración, a través del relato que describía cómo una profesora de asignatura cuestionaba el trabajo de una profesora de apoyo en función de los pocos avances que observaba de sus estudiantes, sin considerar las dificultades específicas. No obstante, resulta pertinente destacar una situación más crítica, que muestra cómo una profesora es inculpada de maltrato infantil por una apoderada de su grupo:

Lloraba de impotencia (...) lloré cuando tuve que ir a defenderme a la Corporación, porque a mí lo menos que se me dijo es que yo tenía más o menos que renunciar, que yo con todo esto, con todo esto yo casi iba a perder mi título. (entrevista 8,61, profesora de apoyo, enseñanza básica)

En esta situación la frustración experimentada por la profesora es descrita como impotencia, debido a la gravedad de la acusación y a las repercusiones que esta tuvo en su vida laboral y personal.

\section{Significados Asociados a la Alegría}

Para el caso de alegría, lo que se buscó fue considerar una gama de expresiones sobre el espectro de la experiencia emocional de los docentes, que podrían considerarse como cualitativamente positivas o agradables y que, por tanto, contribuirían a su alegría.

Me alegran los logros de mis estudiantes. De manera complementaria a lo visto en las situaciones donde surge la frustración, la alegría surge producto de constatar ciertos logros en los estudiantes, logros observados directamente por el profesor o bien a través de parámetros formales de evaluación:

Yo siento gran satisfacción cuando veo que un niño ha obtenido logros de aprendizaje, uno se siente muy contenta con eso. (entrevista 5, 102, profesora regular, enseñanza básica)

Pero la evaluación positiva que motiva la alegría en los docentes no solo se remite a eventos puntuales o acotados, sino también es fruto de la estimación de un proceso virtuoso ocurrido a lo largo del tiempo:

Y los avances se ven registrados en las notas [calificaciones], porque ella tiene buenas notas [calificaciones]. Ha superado bastante la nota [calificación] con respecto al año anterior. (entrevista 5, 83, profesora regular, enseñanza básica)

No obstante, no siempre estos logros están referidos a aprendizajes académicos. A veces, la alegría surge de la evaluación positiva que se hace del mejoramiento observado en el comportamiento de los estudiantes. Además, dicha evaluación no necesariamente se realiza a partir de cánones sobre lo que debe ser o no un buen comportamiento en el contexto escolar, sino más bien considerando lo que resulta conveniente para el contexto social más amplio:

Yo me he sentido súper, me agrada, me siento como más relajada de saber que el niño va a poder salir adelante, ver que está teniendo conductas que le van a poder permitir sobrevivir, porque este mundo para mí es de repente un poquito como la selva. (entrevista 5, 261, profesora de apoyo, enseñanza básica)

De manera especial, destaca el aprendizaje logrado por un estudiante, cuyas dificultades en lectoescritura hacían pronosticar a su profesora jefe una repitencia de curso, pero que, gracias al esfuerzo coordinado y 
sistemático entre su madre y la profesora de apoyo, logran un avance destacable en dicha área, utilizando un material didáctico específico:

Y un día el niño llega con sus tarjetas aprendidas, que era de sílabas directas con cuatro consonantes (...) yo me sentí súper contenta (entrevista 6, 207, profesora de apoyo, enseñanza básica)

En este sentido, la alegría es significada no solo a partir del resultado de aprendizaje alcanzado por el estudiante, sino también por las condiciones distintivas del contexto en que este surge, caracterizado por un importante nivel de dificultad que los protagonistas debieron afrontar recurriendo a un nivel destacable de esfuerzo y sistematicidad.

Fue difícil pero el resultado fue satisfactorio. Tal como se vio anteriormente, es importante destacar que cuando los logros van precedidos de dificultad, esta es significada como un elemento distintivo que despierta satisfacción. Es importante destacar que, si bien la experiencia de dificultad no cabe dentro de la categoría de emoción, bien podría calificarse como una experiencia afectiva no agradable y, por tanto, bajo este punto de vista, resulta curioso el hecho de que esta active emociones de valencia positiva en los profesores.

En concreto, se hace alusión al logro pedagógico alcanzado por un estudiante que mostraba dificultades importantes y que debía enfrentarse por primera vez al desafío de leer y comprender un texto literario. Además, existía un gran cuestionamiento a que dicho estudiante continuara en educación regular e ingresara a enseñanza media, debido a tales dificultades y donde, pese a ello, la profesora de apoyo asumió de manera protagónica el desafío, aunque también dudando de las reales posibilidades del estudiante. Sin embargo, gracias al esfuerzo sistemático y pese a las resistencias contextuales, el joven finalmente logra un nivel básico de aprendizaje:

Fue difícil, pero el resultado yo creo que fue como, de experiencia para el alumno, fue satisfactorio (...) Sin embargo, yo vi que el alumno tuvo como su primera experiencia, primer encuentro, con esto de hojear un libro, de leerlo, de saber que algo pasaba, que había una historia que tenía principio y tenía fin, que eso lo podría relacionar con su vida... Yo creo que ese es como un, como un episodio que tiene que ver más con, con... con satisfacción, que empieza con dudas, pero luego con satisfacción. (entrevista 1, 76, profesora de apoyo, enseñanza media.

Me siento contenta y satisfecha cuando salgo de una clase que me resultó. Pero las sensaciones positivas no provienen solo de los buenos resultados luego de un largo proceso de trabajo, sino también del propio éxito cotidiano, de aquel que se logra día a día. En particular, la sensación de satisfacción emerge a partir de la percepción de que una clase haya provocado un efecto positivo en los propios estudiantes, lo que refleja, por una parte, el lugar central que tiene el aprendizaje de los estudiantes en el quehacer del docente y, por otra, la íntima interdependencia entre el propio comportamiento del docente y los efectos de este sobre los estudiantes:

Me siento muy contenta cuando salgo de una clase muy motivada y que generé un click, salgo ¡ahh! [expresión de satisfacción] esa es mi satisfacción. (entrevista 7, 107, profesor regular, enseñanza básica)

Sentí una alegría enorme porque compatibilizamos inmediatamente. Desarrollar un trabajo conjunto en sala con otro colega es un desafío que se podría catalogar como difícil e incierto. Por ello, antes de que ocurra un primer encuentro entre docentes con distintos roles (duplas), surgen preocupaciones sobre las eventuales diferencias de personalidad o formas de trabajo, que se podrían transformar en obstáculos para lograr un trabajo colaborativo. En ese sentido, la experiencia de sintonía y de afinidad interpersonal es experimentada muy positivamente:

E1: (...) ¿qué otra emoción te evocó, qué sentiste además de... cuando tuviste que asumir esta nueva tarea?

ER: Alegría, po... Una alegría enorme (...) compatibilizamos inmediatamente, nuestros caracteres (...) y derribamos todas esas barreras, entonces, hubo afinidad, afinidad en todo sentido. (entrevista 1, 378, profesora regular, enseñanza media)

Soy feliz bajo ciertas condiciones de trabajo. Algunas características del contexto educativo fueron significadas como relevantes para comprender el surgimiento de emociones de agrado en las profesoras, tales como el ciclo escolar al que han sido asignadas o la calidad de las relaciones que se dan entre los colegas. Una de estas características es la cantidad de estudiantes por sala de clases, lo cual permitiría realizar ciertas actividades pedagógicas que bajo contextos de aulas más numerosas serían más difíciles de realizar:

Yo era feliz con el curso de 24 niños del año pasado, ese. Claro, po, si tú tienes 20 en una sala, eh, yo, por ejemplo, con el $5^{\circ} \mathrm{B}$ trabajo más o menos bien ¿por qué? porque son 24 . He hecho cosas que no he hecho con ningún otro curso con ese curso. Eso cambia mucho las cosas. (entrevista 6, 166, profesora regular, enseñanza básica) 


\section{Discusión}

A continuación se discuten los resultados en función de las diversas inquietudes que han alimentado esta investigación, su contribución en la construcción de la realidad que se investiga y el aspecto axiológico que las acompaña.

La consecución de logro pedagógico y adaptación del comportamiento al contexto escolar estuvo presente en los relatos referidos a eventos donde se experimentaba frustración y alegría, hecho que encuentra resonancia en la conexión entre significados, emociones y realidad socialmente construida que es posible desprender del planteamiento de Bruner (1990/1991). Si bien es cierto que las emociones tienen una base biológica reconocida (Damásio, 1994/1999), el condicionamiento que la cultura les imprime las transforma, a su vez, en parte constitutiva de la propia interpretación del mundo (Bruner, 1985/1996; Mascolo et al., 2003). En este sentido, resulta indicativo que el grado de ajuste o desajuste a parámetros propios del sistema escolar haya estado en el centro de los significados referidos a alegría y frustración, es decir, emociones cuya aparición es posible de comprender como consecuencia de haber conseguido o no el logro de determinados objetivos (Chóliz, 2005).

Los resultados permitirían comprender con mayor detalle el efecto que imprime la cultura escolar sobre las emociones de los docentes, las cuales, a su vez, mediante las disposiciones e indisposiciones que estas expresan a los demás actores, actuarían reforzando la construcción de dicha realidad escolar. Esto se puede notar con mayor claridad en aquellos casos en que las emociones referidas por los docentes surgían en relación directa al comportamiento de un estudiante. Tal como se podría esperar, en todas esas referencias hay coherencia entre la valencia de la emoción que el docente experimenta y la evaluación que dicho profesor realiza sobre el comportamiento del actor aludido (p. ej., "Me da rabia que no se comporten como se debe" o "Me alegran los logros de mis estudiantes"). Asimismo, este tipo de situaciones permite imaginar cómo es que las emociones funcionan discursivamente (Zembylas, 2005) e informan en tiempo real a los estudiantes de un determinado parámetro de la propia realidad subjetiva de sus profesores, la que, a su vez, ha sido modelada por la propia institucionalidad educativa. Dicho proceso permitiría reforzar en los estudiantes, generalmente de manera implícita y mediante diversos marcadores corporales (Konar \& Chakraborty, 2015; Watzlawick, Bavelas \& Jackson, 1967/2002), la realidad relacional que caracteriza al sistema educativo y que, como ya se ha señalado, enfatiza decididamente el logro pedagógico y un comportamiento escolar acorde.

Sin embargo, este ensalzamiento del éxito y el fracaso escolar, lejos de garantizar buenos resultados, tal como intuitivamente podría suponerse, ha sido cuestionado por autores como Hargreaves (2003), quien ha destacado las consecuencias de políticas emocionales centradas en el fracaso de las escuelas y que básicamente terminan reforzando el fracaso escolar y la desigualdad social. Perrenoud (1990/1996) pone su acento en la fabricación de jerarquías de excelencia escolar, minuciosamente diseñadas, a través de sistemas de evaluación, reforzando con ello las desigualdades sociales. Por su parte, Terigi (2009) destaca cómo las categorías de éxito y fracaso escolar reflejan, más que condiciones individuales, el énfasis homogenizador propio del sistema escolar. A lo anterior habría que agregar la crítica realizada a la orientación al éxito individual que imprimen las actuales políticas públicas a la educación, materializadas en procesos tales como la rendición de cuentas o la medición estandarizada de los resultados (Cornejo et al., 2015; Sisto \& Fardella, 2014). En un plano más específico, dado que las categorías éxito y el fracaso escolar permean significativamente la actividad educativa, es posible estimar un efecto obstaculizador sobre la valoración de las diferencias en el quehacer del aula. Bajo esta perspectiva, el funcionamiento binario que se configura a partir de ambas categorías actúa muy posiblemente como un impedimento para apreciar la amplia variedad de potencial existente en los estudiantes. Una práctica pedagógica inclusiva exigiría por parte de sus agentes de una disposición caleidoscópica que permita valorar las diversas situaciones que ocurren en la interacción que se da en el espacio escolar, lo cual, evidentemente, debiera traducirse en una ampliación de las posibilidades relacionales que es posible abrir, en comparación a una sensibilidad restringida a solo dos valores posibles.

La concepción individualista del éxito y fracaso escolar colisiona fuertemente con las orientaciones que buscan una cultura inclusiva, dado que esta requiere de un fuerte sentido de comunidad y cooperación. El establecimiento de lazos de colaboración y apoyo constituye una de las características centrales de la educación inclusiva, lo cual incluso ha llevado a señalar el aprendizaje entre iguales como unas de las estrategias más recomendadas para lograr aquello. Es decir, desde esta perspectiva, no solo se tiene en mente el logro de determinados aprendizajes de contenidos, sino que, sobre todo, se visualiza como fundamental la 
relación de colaboración entre iguales como estrategia para el desarrollo de aprendizajes de naturaleza social y relacional (Duran, 2009).

De manera más específica, los resultados muestran que las emociones de los docentes y los significados que las impregnan surgen en relación tanto a la propia experiencia individual como en relación al contexto. Respecto de esto último, fue posible hallar significados que apuntaban a distintos niveles de la organización escolar para comprender las emociones docentes. Un primer nivel se refería a la interacción directa con estudiantes, colegas y apoderados; un segundo nivel se relacionaba con los estamentos superiores del establecimiento, con los cuales el contacto cotidiano y directo era más discreto y, finalmente, un tercer nivel apuntaba a instancias educativas que operan más allá de los límites de la escuela. En relación al primer nivel, destacan las diferencias de criterio entre un docente regular y otro de apoyo para evaluar los avances pedagógicos con estudiantes o bien se destacan como relevantes las sensaciones de alegría asociadas al alto grado de afinidad y entendimiento entre una profesora regular y una profesora de apoyo. Ambas situaciones reflejarían la importancia, reportada desde la práctica pedagógica, de generar las condiciones básicas para lograr un trabajo colaborativo entre profesionales. En un nivel inmediatamente superior, es destacable la frustración surgida en docentes regulares y de apoyo que trabajaban conjuntamente, a propósito de la transgresión realizada por parte de autoridades escolares al principio básico de participación, situación que pone de manifiesto el valor del liderazgo para lograr los objetivos deseados. Finalmente, en un nivel aún más amplio, es destacable la frustración surgida en los profesores producto de la falta de mutualidad percibida en la relación existente entre la Corporación Municipal y el establecimiento, cuestión que remite, a su vez, a la necesidad de desarrollar entre todos los actores un tipo de relación caracterizada por el respeto y la justicia. Por último, cabe mencionar la similitud entre los niveles observados en este estudio y los niveles que se pueden observar en el modelo propuesto por Fried, Mansfield y Dobozy (2015) para describir los factores que, o bien ejercen influencia sobre las emociones de los docentes, o bien reciben la influencia de estas: intrapersonal, interpersonal o social y cultural y político.

A su vez, cabe destacar los aspectos axiológicos que surgieron del análisis realizado (Echeita et al., 2013; Urbina Hurtado, 2013; Urquhart, 2009), reflejados no solo en la noción de injusticia, aludida explícitamente en uno de los episodios, sino también en cada una de las valoraciones que tácitamente están presentes en las experiencias emocionales relatadas por los actores. Dichas experiencias de agrado o desagrado (Barrett \& Russell, 2015) muy probablemente contribuyen a configurar una determinada ética del espacio educativo, la cual, a su vez, propiciaría el surgimiento de un determinado tipo de convivencia, nutriendo o intoxicando, en definitiva, la experiencia de aprender y enseñar. Efectivamente, en congruencia con autores que desde la filosofía relevan el aspecto evaluativo de las emociones (Nussbaum, 2006; Solomon, 2006/2007), es en el ámbito de lo emocional por sobre las declaraciones manifiestas donde más posiblemente sea factible encontrar los códigos éticos que se transan y efectivamente dan vida o no a una práctica inclusiva.

Finalmente, es importante destacar el hecho de que algunas experiencias afectivas de valencia negativa, luego de un proceso reflexivo, fueran significadas y experimentadas como experiencias positivas. Lo anterior, bien podría asociarse a discursos o narrativas en torno a la identidad del docente y a un deber ser, comprensibles a partir de aspectos como la vocación, el servicio o el sacrificio (Tenti Fanfani, 2006). Sin embargo, también es factible que lo anterior se explique desde la valoración, realizada a partir de la propia experiencia, respecto de la contribución que aspectos como el esfuerzo, la frustración o incluso el sufrimiento pueden tener, bajo ciertas circunstancias, sobre el desarrollo personal (Naranjo, 2005).

\section{Conclusiones}

Tanto desde la evidencia como desde la reflexión, existe el reconocimiento de que la textura de la dimensión afectiva del trabajo educativo anida una serie de cualidades que son fundamentales para lograr una transformación que permita una educación más inclusiva. Este proceso de cambio exige no solo pensar la educación de manera distinta, sino también de sentirla de una forma diferente (Echeita et al., 2013). De ahí que en este trabajo se haya interrogado a la realidad social de espacios educativos que han asumido explícitamente esta tarea, investigando los significados que intercambian sus docentes a partir de la propia experiencia emocional. Este estudio ha permitido explorar también la conexión entre dichas emociones y los significados y, por ende, la realidad social que allí se construye.

Por otra parte, es relevante observar cómo el espacio de lo emocional permite apreciar con claridad la presencia de la dimensión axiológica de la educación y en el que significados relacionados con la injusticia y la mutualidad surgen desde las propias emociones reportadas. 
Cabe destacar el potencial encontrado en el análisis de las emociones de los profesores para la comprensión de la complejidad de las distintas aristas y niveles del trabajo orientado a dar respuesta a la diversidad. La naturaleza espontánea, experiencial y situada de estos estados mentales podría ser de utilidad para generar una manera diferente de comprender y abordar esta tarea. Asimismo, es destacable que el carácter crítico de muchos de los episodios analizados bien podría explicarse como fruto de la cultura escolar prevaleciente, caracterizada por su predominante orientación al éxito individual. En este sentido, es importante destacar la necesidad de fortalecer liderazgos educativos que promuevan, garanticen y, sobre todo, pongan en práctica el principio de participación como principio rector de la educación. Asimismo, es posible estimar que un trabajo centrado en el análisis reflexivo de la gestión de las emociones de los actores educativos ante situaciones críticas podría constituirse en una herramienta interesante de desarrollar como recurso que contribuya a dar respuesta a la diversidad. También es destacable el poder transformador de la experiencia emocional que emerge del análisis realizado, en términos de que emociones experimentadas inicialmente como negativas pueden llegar a impulsar la movilización de recursos personales para afrontar dichas situaciones críticas.

Finalmente, es necesario señalar las limitaciones del trabajo realizado, en cuanto que se trata de un análisis secundario de un material cuyo foco no fue la profundización sobre las emociones experimentadas por los docentes ni de los significados involucrados en dicha vivencia. En ese sentido, considerando la riqueza de los datos obtenidos bajo estas circunstancias, resulta recomendable realizar trabajos dirigidos a ahondar en este tipo de estados mentales en el trabajo de aulas enfrentadas a crecientes niveles de complejidad y diversidad, tanto en relación a la fenomenología involucrada como en relación a aspectos como las creencias, concepciones y significados asociados a esta, y los diversos niveles relacionales implicados al interior de la escuela.

\section{Referencias}

Aguado, L. (2005). Emoción, afecto y motivación. Madrid, España: Alianza.

Albornoz, N., Silva, N. \& López, M. (2015). Escuchando a los niños: significados sobre aprendizaje y participación como ejes centrales de los procesos de inclusión educativa en un estudio en escuelas públicas en Chile. Estudios Pedagógicos, 41( ${ }^{\circ}$ Especial), 81-96. https://doi.org/10.4067/S0718-07052015000300006

Barrett, L. F. \& Russell, J. A. (2015). The psychological construction of emotion. New York, NY: Guilford Press.

Belli, S. (2009). La construcción de una emoción y su relación con el lenguaje: revisión y discusión de una área importante de las ciencias sociales. Revista Theoria: Ciencia, Arte y Humanidades, 18(2), 15-42. Extraído de http://www.ubiobio.cl/miweb/webfile/media/194/v/v18-2/03.pdf

Bruner, J. (1985/1996). Realidad mental y mundos posibles: los actos de la imaginación que dan sentido a la experiencia (B. López, Trad.; Título original: Actual minds, posible worlds). Barcelona, España: Gedisa.

Bruner, J. (1990/1991). Actos de significados: más allá de la revolución cognitiva (J. C. Gómez \& J. Linaza, Trads.; Título original: Acts of meaning). Madrid, España: Alianza.

Chóliz, M. (2005). Psicología de la emoción: el proceso emocional. Valencia, España: Universitat de València. Extraído de https://www.uv.es/=choliz/Proceso\%20emocional.pdf

Cornejo, R., Albornoz, N., Castañeda, L., Palacios, D., Etcheberrigaray, G., Fernández, R. ... Lagos, J. I. (2015). Las prescripciones del trabajo docente en el nuevo marco regulatorio de políticas educativas en Chile. Psicoperspectivas, 14(2), 72-83. https://doi.org/10.5027/psicoperspectivas-Vol14-Issue2-fulltext-580

Damásio, A. (1994/1999). El error de Descartes: la razón de las emociones (3a ed.; P. Jaccomet, Trad.; Título original: Descartes' error: Emotion, reason and the human brain). Santiago, Chile: Andrés Bello

Duran, D. (2009). El aprendizaje entre alumnos como apoyo a la inclusión. En C. Giné (Coord.), La educación inclusiva. De la exclusión a la plena participación de todo el alumnado (pp. 95-109). Barcelona, España: Horsori.

Echeita, G., Sandoval, M. \& Simón, C. (2016, Marzo). Notas para una pedagogía inclusiva en las aulas. Ponencia presentada en el IV Congreso Iberoamericano sobre el Síndrome de Down, Salamanca, España. Extraído de http://cddown-inico.usal.es/docs/045.pdf

Echeita, G., Simón, C., López, M. \& Urbina, C. (2013). Educación inclusiva. Sistemas de referencia, coordenadas y vórtices de un proceso dilemático. En M. A. Verdugo Alonso \& R. L. Schalock (Coords.), Discapacidad e inclusión: manual para la docencia (pp. 329-357). Salamanca, España: Amarú.

Ekman, P., Friesen, W. V. \& Ellsworth, P. (1982). What emotion categories or dimensions can observers judge from facial behavior? En P. Ekman (Ed.), Emotion in the human face (2a ed., pp. 39-55). New York, NY: Cambridge University Press.

Flick, U. (2002/2007). Introducción a la investigación cualitativa (T. del Amo, Trad.; Título original: Qualitative Forschung: Theorie, Methoden, Anwendung in Psychologie und Sozialwissenschaften). Madrid, España: Morata.

Flores, R. \& Naranjo, C. (2013). Análisis de datos cualitativos: el caso de la grounded theory (teoría fundamentada). En M. Canales (Coord.), Escucha de la escucha: análisis e interpretación en la investigación cualitativa (pp. 75-113). Santiago, Chile: Instituto de Estudios Internacionales/LOM.

Fried, L., Mansfield, C. \& Dobozy, E. (2015). Teacher emotion research: Introducing a conceptual model to guide future research. Issues in Educational Research, 25, 415-441. Extraído de http://www.iier.org.au/iier25/fried.pdf

Golombek, P. \& Doran, M. (2014). Unifying cognition, emotion, and activity in language teacher professional development. Teaching and Teacher Education, 39, 102-111. https://doi.org/10.1016/j.tate.2014.01.002

Hargreaves, A. (2000). Mixed emotions: Teachers' perceptions of their interactions with students. Teaching and Teacher Education, 16, 811-826. https://doi.org/10.1016/S0742-051X(00)00028-7

Hargreaves, A. (2003). La política emocional en el fracaso y el éxito escolar. En Á. Marchesi \& C. Hernández Gil (Coords.), El fracaso escolar: una perspectiva internacional (pp. 229-254). Madrid, España: Alianza. 
Izard, C. E. (1977). Human emotion. New York, NY: Springer. https://doi.org/10.1007/978-1-4899-2209-0

Kamenetzky, G. V., Cuenya, L., Elgier, A. M., López Seal, F., Fosacheca, S., Martin, L. \& Mustaca, A. E. (2009). Respuestas de frustración en humanos, Terapia Psicológica, 27, 191-201. https://doi.org/10.4067/S0718-48082009000200005

Konar, A. \& Chakraborty, A. (2015). Emotion recognition: A pattern analysis approach. Hoboken, NJ: John Wiley \& Sons.

López Cruz, M., Valdivia Barrios, A. \& Fernández Droguett, R. (2016). Producciones narrativo-visuales y voz de los y las estudiantes: indagación sobre los significados de participación en niños, niñas y jóvenes en escuelas municipales en Chile. Forum: Qualitative Social Research, 17(1), Artículo 4. Extraído de http://www.qualitative-research.net/index.php/fqs/article/viewFile/2244/3911

López, M., Echeita, G. \& Martín, E. (2010). Dilemas en los procesos de inclusión: explorando instrumentos para una comprensión de las concepciones educativas del profesorado. Revista Latinoamericana de Educación Inclusiva, 4(2), 155-176. Extraído de http://www.rinace.net/rlei/numeros/vol4-num2/art8.pdf

López, V., Julio, C., Morales, M., Rojas, C. \& Pérez, M. V. (2014). Barreras culturales para la inclusión: políticas y prácticas de integración en Chile. Revista de Educación del Ministerio de Educación, Cultura y Deporte de España, 363, $256-281$. https://doi.org/10.4438/1988-592X-RE-2012-363-180

Mascolo, M. F., Fischer, K. W. \& Li, J. (2003). Dynamic development of component systems of emotions: Pride, shame, and guilt in China and the United States. En R. J. Davidson, K. R. Scherer \& H. H. Goldsmith (Eds.), Handbook of affective sciences (pp. 375-408). New York, NY: Oxford University Press.

Miles, M. B. \& Huberman, A. M. (1994). Qualitative data analysis: An expanded sourcebook (2a ed.). Thousand Oaks, CA: SAGE.

Muñoz Villa, M. L., López Cruz, M. \& Assaél, J. (2015). Concepciones docentes para responder a la diversidad: ¿barreras o recursos para la inclusión educativa? Psicoperspectivas: Individuo y Sociedad, 14(3), 68-79. https://doi.org/10.5027/psicoperspectivas-Vol14Issue3-fulltext-646

Naranjo, C. (2005). Cambiar la educación para cambiar el mundo (2a ed.). Santiago, Chile: La Llave.

Norwich, B. (2014). Recognising value tensions that underlie problems in inclusive education. Journal of Applied Statistics, $44,495-510$. https://doi.org/10.1080/0305764X.2014.963027

Nussbaum, M. (2006). El ocultamiento de lo humano: repugnancia, vergüenza y ley. Buenos Aires, Argentina: Katz.

Oatley, K. (1992). Best laid schemes: The psychology of emotions. New York, NY: Cambridge University Press.

Perrenoud, P. (1990/1996). La construcción del éxito y del fracaso escolar (2ª ed.; P. Manzano, Trad.; Título original: La fabrication de l'excellence scolaire : Du curriculum aux practiques dévaluation). Madrid, España: Morata.

Polou, M. \& Norwich, B. (2002). Cognitive, emotional and behavioural responses to students with emotional and behavioural difficulties: A model of decision-making. British Educational Research Journal, 28, 111-138. https://doi.org/10.1080/01411920120109784

Sisto, V. \& Fardella, C. (2014). El eclipse del profesionalismo en la era de la rendicion de cuentas - modelando docentes en el contexto del nuevo management público. Cadernos de Educação, 49, 3-23. https://doi.org/10.15210/CADUC.V0I49.5580

Slee, R. (2011/2012). La escuela extraordinaria: exclusión, escolarización y educación inclusiva (M. Berández, Trad.; Título original: The irrregular school: Exclusion, schooling and inclusive education). Madrid, España: Morata.

Solomon, R. C. (2006/2007). Ética emocional: una teoría de los sentimientos (P. Hermida, Trad.; Título original: True to our feelings: What our emotions are really telling us). Barcelona, España: Paidós.

Strauss, A. \& Corbin, J. (1998/2002). Bases de la investigación cualitativa: técnicas y procedimientos para desarrollar la teoría fundamentada (E. Zimmerman, Trad.; Titulo original: Basics of qualitative research: Techniques and procedures for developing grounded theory). Medellín, Colombia: Editorial Universidad de Antoquia.

Tenti Fanfani, E. (2006). Profesionalización docente: consideraciones sociológicas. En E. Tenti Fanfani (Comp.), El oficio de docente: vocación, trabajo y profesión en el siglo XXI (pp. 119-142). Buenos Aires, Argentina: Siglo XXI.

Terigi, F. (2009). Las trayectorias escolares: del problema individual al desafío de política educativa. Buenos Aires, Argentina: Ministerio de Educación/Organización de los Estados Americanos. Extraído de http://www.bnm.me.gov.ar/giga1/documentos/EL004307.pdf

Torres, N., Lissi, M. R., Grau, V., Salinas, M., Silva, M. \& Onetto, V. (2013). Inclusión educativa: componentes socio-afectivos y el rol de los docentes en su promoción. Revista Latinoamericana de Educación Inclusiva, 7(2), 159-173. Extraído de http://repositoriocdpd.net:8080/handle/123456789/1870

Urbina Hurtado, C. (2013). La compleja relación entre las concepciones sobre los procesos de inclusión y la práctica docente (Tesis de Doctorado, Facultad de Psicología y Facultad de Formación de Profesorado y Educación, Universidad Autónoma de Madrid, España). Extraído de https://repositorio.uam.es/bitstream/handle/10486/13041/62531_Urbina\%20Hurtado\%20Carolina.pdf?sequence=1

Urquhart, I. (2009). The psychology of inclusion: The emotional dimension. En P. Hick, R. Kershner \& P. T. Farrell (Eds.), Psychology for inclusive education: New direction in theory and practice (pp. 66-78). New York, NY: Routledge.

Watzlawick, P., Bavelas, J. B. \& Jackson, D. D. (1967/2002). Teoría de la comunicación humana: interacciones, patologías y paradojas (12 ed.; N. Rosenblatt, Trad.; Título original: Pragmatics of human communication: A study of interactional patterns, pathologies and paradoxes). Barcelona, España: Herder.

Zembylas, M. (2005). Discursive practices, genealogies, and emotional rules: A poststructuralist view on emotion and identity in teaching. Teaching and Teacher Education, 21, 935-948. https://doi.org/10.1016/j.tate.2005.06.005

Zenteno-Osorio, S. \& Leal-Soto, F. (2016). Los afectos en la experiencia de ser profesor de un estudiante diagnosticado con trastorno espectro autista. Pensamiento Educativo, 53(1), 1-14. https://doi.org/10.7764/PEL.53.1.2016.5

Fecha de recepción: Diciembre de 2016.

Fecha de aceptación: Octubre de 2018. 